\title{
Health Information-Seeking Behavior of Filipino University Students: Correlates of their Intolerance of Uncertainty and Cyberchondria
}

\author{
John Paul A. Banzon, Ian Christopher N. Rocha, Dennise C. Cariaso, Kenneth C. Nieveras, \\ Khirsna Kirstine H. Pasajol, Ma. Ann Miyel M. Sigui, Sydney Angela V. Valdez, \\ Hana Mae Kornelia B. Vargas \\ School of Medicine, Centro Escolar University, Manila, Philippines
}

\begin{abstract}
In this modern world where free medical information can be accessible with just one click away, being bombarded with such information may develop a new anxiety called cyberchondria. The study aims to identify the relationship between intolerance of uncertainty and cyberchondria. This phenomenon will be studied as how it affects a person and perceives to uncertain situations prior to testing the respondents' degree of cyberchondria. This study involved students of a private university in the Philippines who were currently enrolled for the school year 2018 to 2019 and who belong to the 18 to 25 age group. A two-stage cluster random sampling method was used to ensure proper representation of subgroups. The results were interpreted using statistical analysis. The findings revealed that there is a significant relationship between intolerance of uncertainty and cyberchondria. The anticipated outcome of this study may be useful in gaining new information regarding cyberchondria and intolerance of uncertainty in order to come up with better management and treatment.
\end{abstract}

\section{Introduction}

In this era of digital revolution, myriad of information is now conveniently accessible in just a few mouse clicks via the internet. The innovation of technology has revolutionized human lives, such as the way in which health information is gained and propagated. For most people, the internet has become the first stop in accessing health-related information due to widespread access of information relating to health and illness with the advantage of convenience, affordability, interactivity and anonymity.

Seeking information about related medical condition on the internet is ubiquitous and often serves beneficial in dealing uncertainty. Although all people face uncertainty and dealing with it is inevitable part of life, there is variability in how individuals cope with its presence. While most individuals feel, empowered and reassured by access to health information and less apprehensive after subsequent pursuance of health information through the internet. Conversely, some individuals became overly distressed and anxious about their health, leading to excessive health-related searches, which only amplify their anxiety. Some studies relate repeated and frequent searches for medical information on the internet to health anxiety and intolerance of uncertainty.

Previous studies have termed this clinical phenomenon when an individual experience unsubstantiated exacerbation of health anxiety as a result of reviewing medical information on the internet as cyberchondria. Internet exploration in pursuance to medical information of common and minor symptoms can escalate into inaccurate knowledge that these symptoms are indication of a serious health condition. The knowledge, empowerment and reassurance brought by the internet searches can be considered beneficial, but it is still arguable whether these advantages outweigh the anxiety that can be invoke by these searches. Moreover, medical information on the internet has the potential to lead to greater level of uncertainty and therefore exacerbate health anxiety for individuals [1].

The present research sought to shed further light in the relationship between intolerance of uncertainty and cyberchondria and extend those findings to determine the measure of Intolerance of uncertainty that significantly contribute to the conditions of cyberchondria.

\section{Literature Review}

Intolerance of uncertainty (IU) is defined as a dispositional characteristic that results from a set of negative beliefs about uncertainty and its implications which involves the tendency to react negatively on an emotional, cognitive, and behavioral level to uncertain situations and events [2]. In the last decade, previous studies have recognized IU as a predictor for a wide range of psychopathologies, including anxiety and depression [3], obsessive-compulsive symptoms [4], emotional problems [5], and psychosis [6].

IU also serves as an important predictor of trait level worry and of the tendency to interpret ambiguous situations in a negative manner, and as 
such, is considered to be a cognitive disposition that might confer risk for general anxiety disorder [7]. IU was first delineated for representing cognitive, emotional, and behavioral reactions to uncertainty in everyday life situations, which was focused initially on understanding worry and generalized anxiety disorder [8]. However, recent evidence has shown that IU represents a critical transdiagnostic construct for understanding neuroticism. There is also proof that fearful responses to unknowns, measured as IU, are important for models of emotion, attachment, and personality. Accordingly, the most recent definition states that IU is an individual's dispositional incapacity to endure the aversive response triggered by the perceived absence of salient, key, or sufficient information, and sustained by the associated perception of uncertainty. The broad importance of fearing the unknown and IU led to clinical research studies exploring interventions targeting the IU construct [9].

The literature on IU and its associated psychopathologies is fast growing, providing evidence for cognitive, behavioral and neural correlates of IU [9]. Those with high IU display attentional biases towards uncertain or ambiguous stimuli [10], display abnormal decision-making processes such as an increase in requirement for evidence before making decisions [11], and perform poorer on basic behavioral tasks such as typing [12]. $\mathrm{IU}$ is also referred as a fear for the unknown, recent theoretical papers place IU at the core of psychopathology, in particular for those individuals with anxiety-related disorders [13].

It has been hypothesized more broadly that attachment difficulties may feel a need for certainty and control, and an intolerance for uncertainty, in that insecure attachment styles can cause individuals to perceive the world as a dangerous place, and insecurely attached individuals may not have adequate resources to cope with uncertain events [14]. Studies had aimed to explore the relationship between IU, insecure attachment styles and personality factors in individuals, assessed using online [15].

In a study which examined the relationship of IU and anxiety upon learning about a certain disease, participants were given pamphlets containing information about the disease which can be either contained of a short or long set of messages. Results showed that people who have read more about the disease showed a higher IU than those who have read the shorter version. Also, people who had a higher IU indicated a higher level of anxiety with regards to the known disease, making them more engaged about knowing its specificities and using this as a mechanism to respond with their uncertainties [16].

On the other hand, online health information seeking, known as cyberchondria, has become common behavior despite the benefits of accessible medical information on the internet. A vicious cycle of increased physical health concerns and online medical information seeking has been identified [17]. Despite proposed theoretical models of cyberchondria, there is a dearth of research investigating risk factors for its development. Potential risk factor is IU which the researchers would like to explore further due to its increasing occurrence.

Cyberchondria or cyberchondriasis is a neologism which is derived from the terms cyber, which is related to information technology and virtual reality, and hypochondria, which is a chronic and abnormal anxiety about imaginary symptoms and ailments [17]. Previous studies took an interest in this concept after it was introduced by popular media. The term can be perceived as deceptive but simple, yet it has been defined in several ways. One meaning of cyberchondria is that it is the modern version of hypochondriasis and that, like its terminological predecessor, it is a mental disorder [18]. In another perspective of meanings, cyberchondria refers to the repeated use of the internet to search for healthrelated information, which leads to negative consequences [18]. These views are yet to be accepted by majority of mental health professionals but overall known by the media [19].

The increased access to information via the internet has caused millions of people to conduct online self-diagnosis. The web has provided ease for many people to obtain medical information and become more involved in their own health. Online self-diagnosis, also known as cyberchondria to other studies, refers to health-seeking individuals depending on technology by searching for their symptoms to generate medical diagnoses themselves without the participation of a healthcare professional [17]. Online self-diagnosing is not only easily accessible but can also be cost and time-saving for individuals. Not to mention, those health-seeking individuals who are hesitant and embarrassed to consult someone about their symptoms can gain anonymity by online self- diagnosing [20].

Previous studies also reported the severity of symptoms of cyberchondria. First symptom is checking online for symptom information from up to 1 to 3 hours per day. Health-seeking individuals who experience high illness anxiety spend up to 2 hours on average a day. Second is fear of having several different diseases. Cyberchondriacs who experience high in illness anxiety feared having nearly 5 diseases. Third is checking 3 to 4 times a day. Those who are high in illness anxiety take up more time searching online for information on their symptoms. Fourth is looking online to get symptom information increases the anxiety. Those high in illness who try to reassure themselves by searching online only worsens their conditions [21]. 
The vast resource of medical information on the web seems problematic for health-seeking individuals with high illness anxiety, which is a hidden effective price for using a cost-effective informational source [21]. Next to a variety of advantages, turning to the internet for medical information also carries a load of disadvantages. Some may include wasting money for unnecessary medication, medical language, lack of peer review, inaccurate or misleading information, and riskpromoting messages [20].

In a recent study, statistics on cyberchondria among university students showed that $14.2 \%$ of students utilize the internet in searching health information when experiencing a health problem. Interestingly, $83.7 \%$ access the internet using their mobile phones and one-fourth of students search health information at least once a week [22].

In the Philippines, a previous study found out that Filipinos use internet in seeking health information online, among other reasons such as social networking, creativity and production, entertainment and leisure, and commercial activity. The results revealed that more than one-thirds of Filipino internet users go online to get general health information [23]. In this regard, there are a number of Filipinos who can are cyberchondriacs.

In this study, IU, the independent variable, is defined as a cognitive bias that affects how a person perceives, interprets, and responds to uncertain situations which is predetermined prior to testing respondents for degree of cyberchondria. While cyberchondria, the dependent variable, is defined as the false belief that one is suffering from a disease that was learned about on the internet or on a specific website which will result to anxiety about one's health given upon classification of their degree of IU.

Therefore, this study aimed to determine the relationship between IU and cyberchondria among Filipino university students. Specifically, this study sought to answer the following questions: 1) is there a relationship between IU and cyberchondria among emerging adults; 2) what is the level of IU among them; and 3) what is their level of cyberchondria?

\section{Methods}

This study used a cross sectional research design which aimed to determine the relationship between IU and cyberchondria. The study required both exposure and disease states to occur at one specific time. The study population were then classified according to four states: university students with high $\mathrm{IU}$ and high cyberchondria, high IU and low cyberchondria, low IU and high cyberchondria, and low IU and low cyberchondria.

The participants of this study were 185 students in a private university in Manila, Philippines. The respondents are emerging adults aged 18 to 25 and currently enrolled in the school year of 2018 to 2019.

Permission in conducting a study in every school was sought from the deans of all 10 schools within the university through a letter signed by the researchers and approved by the university. All procedures performed in this study were also approved by the Ethics Review Committee of the university in accordance with the 1964 Helsinki declaration and its later amendments or comparable ethical standards. Informed consent was also obtained from all individual participants included in the study. Included in the consent are the purpose, significance of the study, procedure, risk and benefits from the study, confidentiality of the respondent's information, and their rights as participants.

The sample size was computed using OpenEpi with an estimated population size of 7,000, a confidence level of $95 \%$, and a margin of error of $5 \%$. The result was 183 participants which was exceeded during the data gathering. The researchers included all 10 schools of the university. Simple random sampling was used to select one program from each school. Another set of random sampling was done to identify the set of students who participated in a given class which makes it a twostage cluster random sampling. The participants were then classified depending on the results.

A three-part self-administered questionnaire was used as an instrument to gather the data. The first part dealt with the characteristics of the students. The second part covered the IU of the students by utilizing the 12-item IU Scale (IUS) [24], which consists two factors. The first factor, prospective IU, reflects approach-oriented responses to uncertainty; its seven items assess individuals' desire for predictability, propensity for active information seeking to reduce uncertainty, and preference for knowing what future events entail. The second factor, inhibitory IU, reflects avoidance-oriented responses to uncertainty; its five items assess the degree to which individuals experience reticence and paralysis when faced with uncertainty [25]. The last part measured the cyberchondria of the students by using the 33-item Cyberchondria Severity Scale (CSS) [26], which measures different aspects such as compulsion, distress, excessiveness, reassurance seeking, and mistrust of medical profession.

Regarding data analysis, the researchers interpreted the results using descriptive statistics such as mean, frequency, and percentages to present the data, and inferential statistics such as Chi square to test the association between IU and cyberchondria since both variables are nominal.

\section{Results}

This portion presents the findings of the study. The results, particularly the characteristics of the 
university students as presented in Table 1 , revealed that more than half are female $(66.5 \%)$ and first year university students $(63.8 \%)$, while more than twofifths are 19 years old $(43.8 \%)$.

Table 1. Profile of university students

\begin{tabular}{|c|c|c|}
\hline Profile $(n=185)$ & Frequency & Percentage \\
\hline \multicolumn{3}{|l|}{ Sex } \\
\hline Female & 123 & $66.5 \%$ \\
\hline Male & 62 & $33.5 \%$ \\
\hline \multicolumn{3}{|l|}{ Age } \\
\hline 18 years old & 39 & $21.1 \%$ \\
\hline 19 years old & 81 & $43.8 \%$ \\
\hline 20 years old & 19 & $10.3 \%$ \\
\hline 21 years old & 19 & $10.3 \%$ \\
\hline 22 years old & 12 & $6.5 \%$ \\
\hline 23 years old & 7 & $3.8 \%$ \\
\hline 24 years old & 5 & $2.7 \%$ \\
\hline 25 years old & 3 & $1.6 \%$ \\
\hline \multicolumn{3}{|l|}{ Year Level } \\
\hline First Year & 118 & $63.8 \%$ \\
\hline Second Year & 8 & $4.3 \%$ \\
\hline Third Year & 19 & $10.6 \%$ \\
\hline Fourth Year & 40 & $21.6 \%$ \\
\hline
\end{tabular}

The following findings were revealed using descriptive statistics for the levels of IU and cyberchondria. Table 2 showed how the university students are classified whether they have high or low cyberchondria and high or low IU through their mean scores and standard deviations. Additionally, results also revealed that there are 10 students who experience high IU and high cyberchondria (5.40\%), 18 students who experience high IU but low cyberchondria $(9.73 \%), 17$ students who experience low IU but high cyberchondria (9.19\%), and 158 students who experience low IU and low cyberchondria $(85.41 \%)$, as presented in Table 3 . These findings were compared with the expected values of levels of IU and cyberchondria presented in Table 4. Surprisingly, the values of observed high IU and high cyberchondria and observed low IU and low cyberchondria exceeded their expected values. In contrast, the values of observed high IU but low cyberchondria and low IU but high cyberchondria did not meet their expected values.

Table 2. Mean scores and standard deviations of intolerance of uncertainty and cyberchondria

\begin{tabular}{lcc}
\hline \multicolumn{1}{c}{ Variables } & Mean & SD \\
\hline Level of Intolerance of Uncertainty & 38.88 & 9.03 \\
Level of Cyberchondria & 87.86 & 22.48 \\
\hline
\end{tabular}

Table 3. Observed values for high and low cyberchondria (CC) and high and low intolerance of uncertainty (IU)

\begin{tabular}{lccc}
\hline Observed & High CC & Low CC & Total \\
\hline High IU & 10 & 18 & 28 \\
\hline
\end{tabular}

\begin{tabular}{lccc}
\hline & $(5.40 \%)$ & $(9.73 \%)$ & $(15.14 \%)$ \\
Low IU & 17 & 140 & 157 \\
& $(9.19 \%)$ & $(75.68 \%)$ & $(84.86 \%)$ \\
Total & 27 & 158 & 185 \\
& $(14.59 \%)$ & $(85.41 \%)$ & $(100 \%)$ \\
\hline
\end{tabular}

Table 4. Expected values for high and low cyberchondria (CC) and high and low intolerance of uncertainty (IU)

\begin{tabular}{lccc}
\hline Expected & High CC & Low CC & Total \\
\hline High IU & 4 & 24 & 28 \\
& $(2.16 \%)$ & $(12.97 \%)$ & $(15.14 \%)$ \\
Low IU & 23 & 134 & 157 \\
& $(12.43 \%)$ & $(72.43 \%)$ & $(84.86 \%)$ \\
Total & 27 & 158 & 185 \\
& $(14.59 \%)$ & $(85.41 \%)$ & $(100 \%)$ \\
\hline
\end{tabular}

In summary, Table 5 presented the prevalence rate of the respondents with high IU and high cyberchondria. Out of 185 respondents, 28 of them are with high IU (15.14\%) and 27 of them are with high cyberchondria (14.59\%).

Table 5. Prevalence rate of intolerance of uncertainty (IU) and cyberchondria (CC)

\begin{tabular}{lc}
\hline Prevalence Rate & Percentage \\
\hline University Students with High IU & $15.14 \%$ \\
University Students with High CC & $14.59 \%$ \\
\hline
\end{tabular}

Regarding the correlations, Table 6 showed the computation of the value of Chi square arriving at 11.80668. The computed degree of freedom is 1 . Using an analytic software, the results revealed a pvalue of 0.00059 which is less than the alpha value of 0.05 , thereby rejecting the null hypothesis and accepting the alternative hypothesis that there is a significant direct relationship between intolerance of uncertainty and cyberchondria.

Table 6. Data analysis applying inferential statistics (application of Chi square and comparison of p-value to alpha level)

\begin{tabular}{cc}
\hline $\mathbf{x}^{\mathbf{2}}$ & 11.80668 \\
$\mathbf{d f}$ & $1(\mathrm{r}-1)(\mathrm{k}-1)$ \\
$\mathbf{p}-\mathbf{v a l u e}$ & 0.00059 \\
$\boldsymbol{\alpha}$ & 0.05 \\
\hline
\end{tabular}

\section{Discussion}

Health information-seeking individuals often search for medical information on the web. For some people, this behavior exacerbates health anxiety which is known as cyberchondria [1]. The extensive source of medical and health information on the internet seems to be a problem for cyberchondriacs with intolerance of uncertainty, which is considered as an advantage for them since they utilize a cost- 
effective source of health and medical information [21]. However, searching on the web for health information involves a lot of disadvantages which include wasting money for buying unnecessary medications seen from the web, difficulty in understanding the medical language, inaccurate or misleading information, among others [20]. At present, little is known about this condition.

In this regard, the present study helped fill this gap in the literature and revealed that IU moderated the impact of cyberchondria among Filipino young adults. The findings of this study have proven that there is an increasing number of cyberchondriacs among Filipino university students since the prevalence rate of cyberchondria in this study revealed to be higher from the prevalence rate shown from the recent studies [22].

This study has also determined that there is a significant relationship between IU and cyberchondria based on the findings of the study which is in relation with the findings of previous studies [1] [17]. Thus, the findings confirm that when IU is high, cyberchondria also follows. Conversely, when IU is low, cyberchondria is also low. These findings suggest that health-seeking individuals who have difficulty tolerating uncertainty are most likely to experience cyberchondria.

In this regard, the significance between IU and cyberchondria could be of use to put further focus of treatment to such vulnerable group, which are the emerging adults aged 18 to 25 and among university students which could be predisposed to having cyberchondria. In addition, since cyberchondria and intolerance of uncertainty are just recently added under generalized anxiety disorder [1] [3] in the 5th edition of the Diagnostic and Statistical Manual of Mental Disorders (DSM-5) [27], this study puts further awareness that such illness is existing in the society.

With this study, health care providers may be able to tailor their way of communication with their patients by assessing their levels of cyberchondria and intolerance of uncertainty and making it as a part of their conversation before providing their patients with diagnosis about their current situation, thus decreasing their level of anxiety [15].

\section{Conclusion}

Therefore, this study suggests that cyberchondria is already existing among young adult populations. These health-seeking individuals tend to get every information through the internet regarding their health. The medical information from the web makes it convenient to university students who are nonexperts to conduct their own diagnosis and health assessment based on the limited information about signs, symptoms, and disorder. These undependable amounts of information have potential to increase anxiety and uncertainty in people who has little or no background in medicine or allied health sciences. Health-seeking individuals who rely on the internet regarding their health may get a negative impact to their general wellbeing.

\section{Recommendation}

This study recommends fostering awareness among students in the university and vulnerable populations such as emerging adults aged 18 to 25 . A further study is recommended to fully understand this new behavior.

\section{References}

[1] Fergus, T. A. (2013). Cyberchondria and intolerance of uncertainty: Examining when individuals experience health anxiety in response to Internet searches for medical information. Cyberpsychology, Behavior, and Social Networking, 16(10), 735-739.

[2] Buhr, K., and Dugas, M. J. (2008). The role of fear of anxiety and intolerance of uncertainty in worry: An experimental manipulation. Behaviour Research and Therapy, 47(3), 215-223.

[3] Carleton, R. N. (2014). The intolerance of uncertainty construct in the context of anxiety disorders: Theoretical and practical perspectives. Expert Review of Neurotherapeutics, 12(8), 937-947.

[4] Toffolo, M. B. J., van den Hout, M. A., Engelhard, I. M., Hooge, I. T. C., and Cath, D. C. Uncertainty, checking, and intolerance of uncertainty in subclinical obsessive-compulsive disorder: An extended replication. Journal of ObsessiveCompulsive and Related Disorders, 3(4), 338-344.

[5] Boswell, J. F., Thompson-Hollands, J., Farchione, T. J., and Barlow, D. H. (2013). Intolerance of uncertainty: A common factor in the treatment of emotional disorders. Journal of Clinical Psychology, 69(6), 630-645.

[6] Broome, M. R., Johns, L. C., Valli, I., Wooley, J. B., Tabraham, P., Brett, C., Valmaggia, L., Peters, E., Garety, P. A., and McGuire, P. K. (2007). Delusion formation and reasoning biases in those at clinical high risk for psychosis. British Journal of Psychiatry, 191(51), s38-s42.

[7] Koerner, N., and Dugas, M. J. (2008). An investigation of appraisals in individuals vulnerable to excessive worry: The role of intolerance of uncertainty. Cognitive Therapy and Research, 32, 619-638. 
[8] Mosca, O., and Lauriola, M. C. (2016). Intolerance of uncertainty: A temporary experimentalinduction procedure. PLOS ONE.

[9] Grupe, D. W., and Nitschke, J. B. (2013) Uncertainty and anticipation in anxiety: an integrated neurobiological and psychological perspective. Nature Reviews Neuroscience, 14(7), 488-501.

[10] Dugas, M. J., Hedayati, M., Karavidas, A., Buhr, K., Francis, K., and Phillips, N. A. (2005). Intolerance of uncertainty and information processing: Evidence of biased recall and interpretations. Cognitive Therapy and Research, 29(1), 57-70.

[11] Carleton, R. N., Duranceau, S., Shulman, E. P., Zerff, M., Gonzales, J., and Mishra, S. (2016). Selfreported intolerance of uncertainty and behavioural decisions. Journal of Behavior Therapy and Experimental Psychiatry, 51, 58-65.

[12] Thibodeau, M. A., Carleton, R. N., GomezPerez, L., and Asmundson, G. J. G. (2013). "What if I make a mistake?" Intolerance of uncertainty is associated with poor behavioral performance. Journal of Nervous and Mental Disease, 201(9), 760-766.

[13] Waller G. A. (2008). A 'trans-transdiagnostic' model of the eating disorders: A new way to open the egg? European Eating Disorders Review, 16(3), 165-172.

[14] Bowlby, J. (1988). A secure base: Parent-child attachment and healthy. United States: Basic Books, Perseus Books Group.

[15] Konstantellou, A., Campbell, M., Eisler, I., Simic, M., and Treasure, J. (2011). Testing a cognitive model of generalized anxiety disorder in the eating disorders. Journal of Anxiety Disorders, 25(7), 864-869.

[16] Rosel, N., Knauper, B., Pasqualina, D., Morrison, E., Tabing, R., Feldstain, A., Amsel, R., Mayrand, M. H., Franco, E., and Rosberger, Z. (2010). The impact of intolerance of uncertainty on anxiety after receiving an informational intervention about HPV: A randomized controlled study. Psychology and Health, 25(6), 651-668.

[17] Norr, A., Albanese, B., Oglesby, M., Allan, N., and Schmidt, N. (2015). Anxiety sensitivity and intolerance of uncertainty as potential risk factors for cyberchondria. Journal of Affective Disorders, 174(15), 64-69.
[18] Starcevic, V. (2017). Cyberchondria: Challenges of problematic online searches for healthrelated information. Psychotherapy and Psychosomatics, 86, 129-133.

[19] Fox, S., and Duggan, M. (2013, January). Health Online 2013. Pew Research Center. Retrieved from_http://www.pewinternet.org/2013/01/15/healthonline-2013/ (Access Date: 20 November, 2020).

[20] Hu, Y., and Haake, J. (2015). Search your way to an accurate diagnosis: Predictors of internet-based diagnosis accuracy. Atlantic Journal of Communication, 18(2), 79-88.

[21] Doherty-Torstrick, E. R., Walton, K. E., and Fallon, B. A. (2016). Cyberchondria: Parsing health anxiety from online behavior. Psychosomatics, 57(4), 390-400.

[22] Bati, A. H., Mandiracioglu, A., Govsa, F., and Çam, O. (2018). Health anxiety and cyberchondria among Ege University health science students. Nurse Education Today, 71, 169-173.

[23] Hechanova, M. R. M., and Ortega-Go, R. (2017). The good, the bad and the ugly: Internet use, outcomes and the role of regulation in the Philippines. Electronic Journal of Information Systems in Developing Countries, 63(8), 1-25.

[24] Carleton, R. N., Norton, M. A., and Asmundson, G. J. G. (2007). Fearing the unknown: A short version of the intolerance of uncertainty scale. Journal of Anxiety Disorders, 21, 105-117.

[25] Hale, W., Richmond, M., Bennett, J., Berzins, T., Fields, A., Weber, D., Beck, M., and Osman, A. (2016). Resolving uncertainty about the intolerance of uncertainty scale-12: Application of modern psychometric strategies. Journal of Personality Assessment, 98(2), 200-208.

[26] McElroy, E., and Shevlin, M. (2014). The development and initial validation of the cyberchondria severity scale (CSS). Journal of Anxiety Disorders, 28(2), 259-265.

[27] American Psychiatric Association. (2013). Diagnostic and statistical manual of mental disorders, $5^{\text {th }}$ edition (DSM-5). Arlington, VA, United States: American Psychiatric Publishing.

\section{Acknowledgment}

The authors would like to express their deepest gratitude to Dr. Cynthia R. Nacpil for her guidance, and the Department of Family and Community Medicine of the School of Medicine of Centro 
International Journal of e-Healthcare Information Systems (IJe-HIS), Volume 7, Issue 1, 2020

Escolar University for their support, as well as its Ethics Review Committee for their approval. The authors would also like to thank the School Deans of Centro Escolar University for their assistance in the data collection. 